\title{
Penetrating craniofacial arrow injury
}

\author{
DK Jain, Gaurav Aggarwal, PS Lubana, Sonia Moses \\ Department of Surgery, M.G.M. Medical College and M.Y. Hospital, Indore, Madhya Pradesh-452 001, India
}

\begin{abstract}
Arrow injuries are an extinct form of injury in most parts of the developed world, but are still seen, albeit infrequently in developing countries. Reports of penetrating injuries of the craniofacial region secondary to projectiles are few and far between. The morbidity-free outcome of surgical removal, in case of penetrating arrow injuries, despite the delay in presentation and, moreover, in the emergency surgical practice, are the salient points to be remembered whilst managing such cases, for 'what the mind knows is what the eyes see and what the eyes see is what can be practiced'. We report the case of a patient who was attacked by a projectile fired from a crossbow. Immediate surgery under general anesthesia was required to remove the arrow, with utmost care to avoid any neurovascular compromise to the facial nerve, as well as minimize postoperative complications such as otitis media and subsequent meningitis.
\end{abstract}

Key words: Arrow injury, craniofacial injury, meningitis

DOI: $10.4103 / 0976-3147.63095$

\section{Introduction}

Arrow injuries are an extinct form of injury in most parts of the developed world but are still seen, albeit infrequently in developing countries. Reports of penetrating injuries of the craniofacial region secondary to projectiles are few and far between. We report the case of a patient who was attacked by a projectile fired from a crossbow. Immediate surgery under general anesthesia was required to remove the arrow, with utmost care to avoid any neurovascular compromise to the facial nerve, as well as minimize post operative complications such as otitis media and subsequent meningitis.

\section{Case Report}

A 40-year-old female, of the Bhil community, from Malwa region, presented to the emergency department of our hospital, following an attempted murder, six hours ago, using a crossbow arrow. The attack was due to an old rivalry between two families. At her arrival to the hospital, she was fully conscious and the neurological and systemic physical evaluations were normal, with a Glasgow coma scale of 15/15. The arrow was impacted firmly in her left occipito-temporal region, narrowly missing her cervical spine [Figures 1, 2]. Craniofacial radiographs [Figure 3] and a tomodensitometric examination were performed. The heavy carbon arrow was $20 \mathrm{~cm}$ long and $6 \mathrm{~mm}$ in diameter. The missile had entered posterior to the left ear, and was lodged midway between the cervical spine and left angle of mandible, narrowly missing the neurovascular bundles situated at this level. Her respiration was settled and all her vital parameters were within normal limits, except for mild tachycardia.

She was immediately taken into the operation theater, and under general anesthesia, using nasal intubation; the precariously placed arrow was carefully removed, via adequate dissection from its surrounding structures, with utmost care to avoid any neurovascular damage, in the presence of a neurosurgeon. After removal of the arrow, the local area was irrigated with antiseptic solution, and tissue approximation was completed in two layers [Figure 4].

The postoperative period was uneventful, with the patient being started on antibiotics, analgesics and serratiopeptidase preparation. She had no neuro deficit and was discharged on the fifth postoperative day, without event, and advised regular follow ups.

\section{Discussion}

Arrow injuries are an extinct form of injury in most parts of the developed world but constitute $0.1 \%$ of emergency admissions in the developing world ${ }^{[1]}$ The various 


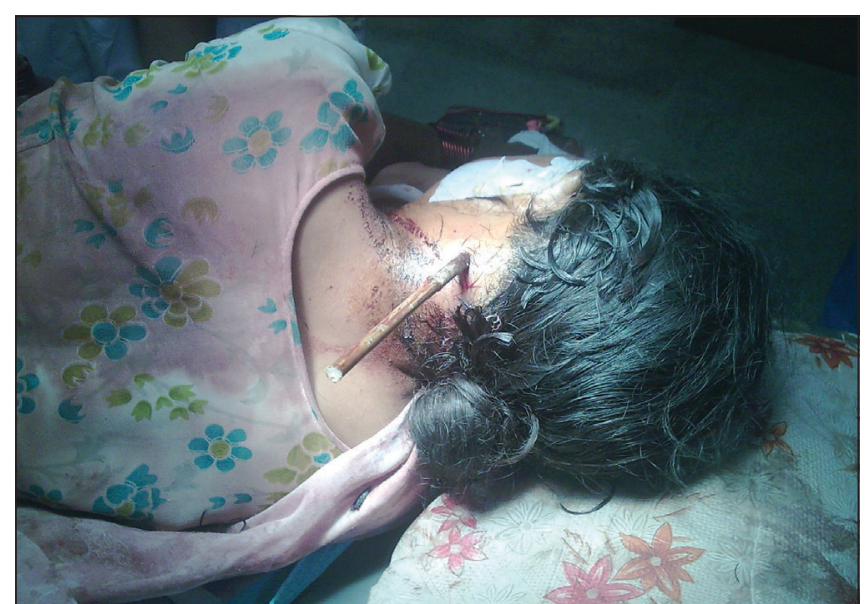

Figure 1: Arrow impacted firmly in the patient's left occipito-temporal region

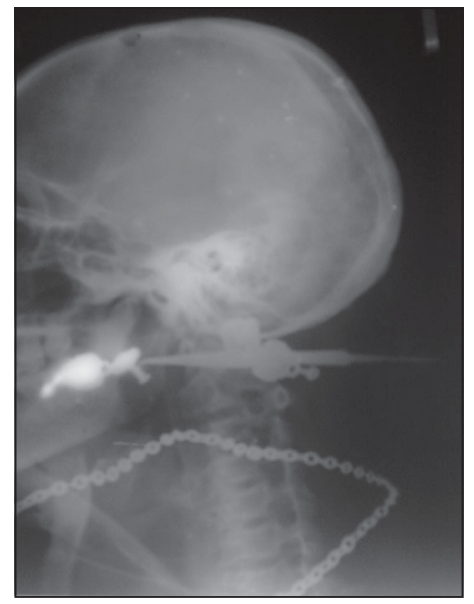

Figure 3: X Ray of the patient: showing the depth of arrow penetration with a bent arrow tip, indicative of the force of impact

reasons for the injuries vary, such as armed robberies in $41 \%$, communal clashes $20.5 \%$, dispute between farmers and cattle herdsmen $13 \%$, and cattle theft $8 \%{ }^{[1]}$

Various factors are responsible for the severity of the injury such as distance of the assailant from the target, the force and trajectory of the arrow, as well as the physical characteristics of the arrow. Despite a relatively low velocity (compared with gunshots), the sharpness and propulsion force of the crossbow may be sufficient to enable penetration of the skull at short range. The major risk is a direct injury of cerebral parenchyma, and a vascular injury is sometimes revealed at the removal of the foreign body. ${ }^{[2]}$

In addition, otitis media and subsequent meningitis following penetrating facial injuries are not uncommon. Post-traumatic meningitis (PTM) can lead to devastating results and mortality rates up to $65 \%$ have been reported. ${ }^{[3-5]}$ While the time between injury and infection

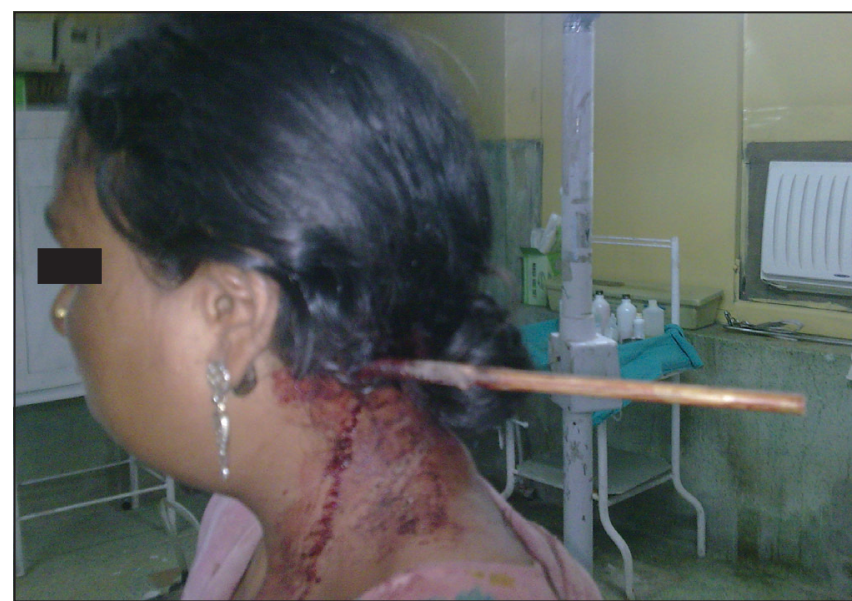

Figure 2: Patient in sitting position: arrow impacted in her left occipito temporal region

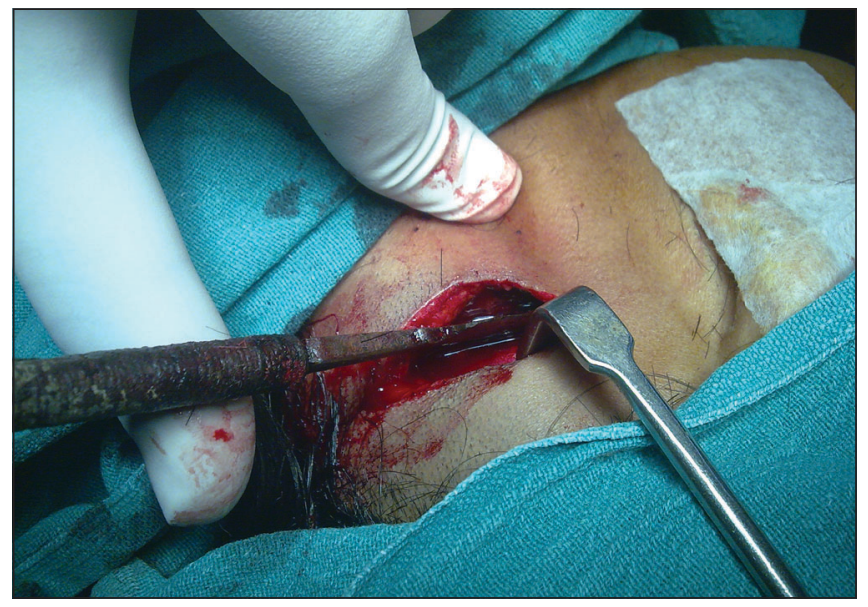

Figure 4: Arrow removal: the residual bed visible beneath

may be brief, there are numerous cases where PTM has been diagnosed years after injury. ${ }^{[6,7]}$ Causative agents for PTM include a wide range of both gram-positive and gram-negative organisms. Streptococcus pneumoniae is the most common agent found in most series and is isolated in $52-100 \%$ of cases. ${ }^{[2]}$

The diagnosis of PTM is usually made by CSF cultures with positive results seen in $73-100 \%$ of patients. Cultures of CSF may fail to yield an isolate, however, and negative cultures have been reported in up to $27 \%$ of cases. ${ }^{[8]}$ Moreover, the facial nerve also lies in close proximity to the site of injury, as in this case, and it is of significance to avoid any injury to it and thus avoid any subsequent neuro deficit. Antibiotics to be given post operatively should be chosen in accordance with the clinical situation and ability to penetrate the blood-brain barrier. Jones found that PTM onset within 3 days in patients with non-penetrating, non-depressed injuries was uniformly pneumococcal and recommend empiric therapy against 
this organism. ${ }^{[8]}$ While the infecting organism is likely to be from the nasopharynx or external auditory canal, nosocomial bacteria cannot be excluded until culture results are known.

In patients with penetrating injuries, prolonged hospitalization, delayed PTM onset, or antibiotic prophylaxis, definitive therapy should include broad spectrum drugs since the risk of infection with gramnegative or resistant microorganisms is higher. ${ }^{[8]}$ Thus, in the management of arrow injuries lies the significance of avoiding any undue time delay, as well as any neurological or vascular compromise, so as to obtain the best possible outcome.

\section{Conclusion}

The morbidity-free outcome of surgical removal, in case of penetrating arrow injuries, despite the delay in presentation and moreover in the emergency surgical practice, are the salient points to be remembered whilst managing such cases, for 'what the mind knows is what the eyes see and what the eyes see is what can be practiced'

\section{References}

1. Madziga AG. Arrow injuries in North East Nigeria. West Afr J Med 2003;22:106-9.

2. Byard RW, Koszyca B, James R. Crossbow suicide: Mechanisms of injury and neuropathologic findings. Am J Forensic Med Pathol 1999;20:347-53.

3. Taha JM, Haddad FS, Brown JA. Intracranial infection after missile injuries to the brain: Report of 30 cases from the Lebanese conflict. Neurosurgery 1991;29:864-8.

4. Appelbaum E. Meningitis following trauma to the head and face. JAMA 1960;173:116-20.

5. Hand WL, Sanford JP. Posttraumatic bacterial meningitis: Ann Intern Med 1970;72:869-74.

6. Wilson NW, Copeland B, Bastian JF. Posttraumatic meningitis in adolescents and children. Pediatr Neurosurg 1990-91;16:17-20.

7. Linell EA, Robinson WL. Head injuries and meningitis. J Neuro Psychiatry 1941;4:23-31.

8. Jones SR, Luby JP, Sanford JP. Bacterial meningitis complicating cranialspinal trauma. J Trauma 1973;13:895-900.

Source of Support: Nil, Conflict of Interest: None declared.

\section{Dispatch and return notification by E-mail}

The journal now sends email notification to its members on dispatch of a print issue. The notification is sent to those members who have provided their email address to the association/journal office. The email alerts you about an outdated address and return of issue due to incomplete/incorrect address.

If you wish to receive such email notification, please send your email along with the membership number and full mailing address to the editorial office by email. 